P-18 AN AUDIT OF THE USE OF ALL-WALES DO NOT ATTEMPT CARDIOPULMONARY RESUSCITATION (DNACPR) ORDERS WITHIN A SPECIALIST PALLIATIVE CARE UNIT (SPCU) IN SOUTH WALES

\author{
${ }^{1,2}$ Anthony Williams. ${ }^{1}$ Cardiff and Vale University Health Board, Cardiff, UK; ${ }^{2}$ Abertawe Bro \\ Morgannwg University Health Board
}

\subsection{6/bmjspcare-2017-00133.18}

Background In 2015, an 'All Wales' DNACPR policy was implemented with the intention of providing consistency and continuation of DNACPR decision making throughout all health care settings within Wales. The aim of the audit was to investigate the use of this policy within a SPCU in Swansea against audit standards outlined in the All Wales DNACPR guidance.

Methods A case note analysis of all patients who died in or were discharged from the SPCU during two 8 week periods between August 2015 and May 2016 was performed. The audit measures included accurate DNACPR form completion, time taken to senior review and wider communication of the DNACPR decision. The audit standard for each measure was $100 \%$. Changes introduced following the first audit included formalisation of the ward clerk role in coordinating DNACPR forms on discharge and use of the handover list to prompt DNACPR form distribution.

Results Adequate completion of DNACPR forms improved from $44 \%$ to $89 \%$ between the two audit periods. Senior review of this decision improved from $81 \%$ to $96 \%$, although mean time to review increased from 1.2 days to 3.8 days. Communication of the DNACPR decision to the GP increased from $77 \%$ to $91 \%$ and communication to the out-of-hours GP increased from $11 \%$ to $73 \%$.

Conclusions This is, to the author's knowledge, the first complete audit cycle in relation to the new All Wales DNACPR policy. Whilst significant improvement was made during completion of the audit cycle further improvements are required to reach the audit standard. Future recommendations include modifying the ward discharge 'check-list' to include the DNACPR form. The audit highlights the challenges of ensuring thorough documentation and dissemination of DNACPR decisions. These results have been shared with the national DNACPR audit which will hopefully influence further evolution of the current policy.

\section{P-19 FAMILY AND CARER INVOLVEMENT IN DECISION MAKING IN PALLIATIVE CARE}

Helen McGee, Sophie Rambihar. Hospice in the Weald, Tunbridge Wells, UK

\subsection{6/bmjspcare-2017-00133.19}

Background Communication with patients and their families/ carers is key to excellent palliative care and both the GMC and NICE state that families/carers should be routinely offered information about their relative within the confines of confidentiality. An audit was conducted at Hospice in the Weald to review the involvement that families/carers had in key decision making for patients on the ward. It reviewed whether all important clinical decisions made whilst the patient was on the ward had been discussed with families/carers

Methods A retrospective case note audit was carried out for all the patients on the ward during one month (September
2015). Information was drawn from the Electronic Care Record (ECR) and paper notes including DNACPR forms. 6 key clinical decisions were identified as instances when a discussion with families/carers would be appropriate. Discussions had to be clearly documented for it to be considered eligible that discussion had taken place.

Results 5/6 (83\%) DNACPR decisions made in the Hospice ward were discussed with families/carers, 30/36 (88\%) families/carers had discussions about ceilings of treatment, 31/32 (97\%) families/carers were involved in discussions regarding medical interventions, $27 / 27$ families/carers (100\%) were told that their relative had deteriorated and 23/23 (100\%) of families/carers had an opportunity to discuss their relatives' end of life needs.

Conclusions These results show that the vast majority of important clinical decisions are communicated with the families and carers of the patients involved however there is room for improvement especially around DNACPR and ceilings of treatment discussions. Often these discussions had been had previously with other healthcare professionals and so were not revisited. This highlights a need for robust shared clinical records and the increased use of EPaCCS to ensure full communication between services.

\section{P-20 A RETROSPECTIVE AUDIT OF BLOOD PRESSURE, ORTHOSTATIC HYPOTENSION AND FALLS IN ADVANCED CANCER}

${ }^{1}$ Amy P Worrall, 'Ciarán Doyle, ${ }^{1}$ Roisín Ní Dhomhnaill, ${ }^{2}$ Declan Walsh. 'School of Medicine, Trinity College Dublin, Dublin 2, Ireland; ' $O u r$ Lady's Hospice and Care Services, Harold's Cross, Dublin

\subsection{6/bmjspcare-2017-00133.20}

Background Orthostatic Hypotension $(\mathrm{OH})$ is a drop of $\geq 20 \mathrm{mmHg}$ systolic blood pressure (BP) and/or diastolic $10 \mathrm{mmHg}$ within 3 minutes of orthostatic stress. ${ }^{1} \mathrm{OH}$ seems highly prevalent in advanced cancer. Comorbidities and antihypertensives increase $\mathrm{OH}$ risk and falls risk. Consequently cancer patients in palliative settings are high fall risks. ${ }^{2}$

Objectives $\mathrm{BP}$ and $\mathrm{OH}$ measurement practices and post-fall interventions were audited amongst in-patients with advanced cancer.

Methods A retrospective analysis of four consecutive months of cancer admissions to a specialist palliative care unit was conducted. Data was obtained from 168 non-randomly selected clinical records. Information recorded included: demographics, falls risk assessment, falls occurrence, BP and relevant medications. The audit was against standards for current institutional clinical policies.

Findings Of 168 admissions, 136 (81\%) had the Falls Risk Screening Tool completed. 143 of them (85\%) had BP recorded, while 25 (15\%) did not. There were 7 falls during the first week post-admission. Post-fall, 5 had BP measured; 2 did not. Only 1 of the 7 who fell had $\mathrm{OH}$ measured.

Conclusions During the audit period none of clinical standards were fully completed. There were 7 falls in one week and only 1 had the required $\mathrm{OH}$ measurement conducted. Some admission tools were misinterpreted or were ambiguous. Review of institutional admission tools could increase compliance and clinical standard adherence, especially if tailored for a palliative care cohort. $\mathrm{OH}$ may be underdiagnosed. 


\section{REFERENCES}

1. Chambers JC. Should we screen hospice inpatients for orthostatic hypotension? PalliativeMedicine 2005;19(4):314-8.

2. Stone CA, Lawlor PG, Kenny RA. How to identify patients with cancer at risk of falling: a review of the evidence. Journal of Palliative Medicine 2011;14;(2):221230 .

\section{P-21 PALLIATIVE MEDICINE PHYSICIANS ARE EXCEEDINGLY OPTIMISTIC INDIVIDUALS!}

${ }^{1}$ Anna Bradley, ${ }^{1}$ Andrew Davies, ${ }^{2}$ Richard Berman, ${ }^{3}$ Louise Mason, ${ }^{4}$ Mark Hill. ${ }^{1}$ Royal Surrey County Hospital, Guildford, UK; ${ }^{2}$ The Christie Hospital, Manchester, UK; ${ }^{3}$ Sussex Cancer Centre, Brighton, UK; ${ }^{4}$ Kent Oncology Centre, Maidstone, UK

10.1136/bmjspcare-2017-00133.21

Background Oncologists (and other healthcare professionals) often perceive palliative medicine physicians as being overly pessimistic, whilst palliative medicine physicians often perceive oncologists as being overly optimistic. However, there appears to be no scientific evidence to support these perceptions. Hence, we set out to examine the relationship between choice of medical specialty and levels of optimism/pessimism.

Method In April 2016 all oncologists and palliative medicine physicians employed at four cancer centres within the United Kingdom were contacted to complete an online survey. Participants were asked to complete the Life Orientation Test Revised (LOT-R), which is a validated measure for assessing optimism and pessimism, and also asked to describe a picture of a partially filled wine glass (ie, would you describe the glass as "half full" or "half empty").

Results 112 participants of different grades (consultant, specialty trainees or "other" doctors) completed the on-line survey in full. There was no difference in LOT-R scores between oncologists and palliative medicine physicians, but there was a statistically significant difference in LOT-R pessimism scores between consultants and specialty trainees $(p=0.03)$. There was also no difference in the glass half full/half empty question between oncologists and palliative medicine physicians, or in this case between consultants and specialty trainees.

Conclusions The results of this study refute the perception that palliative medicine physicians are overly pessimistic (and that oncologists are overly optimistic). Interestingly, specialty trainees were generally less optimistic than consultants, which may reflect the current challenges facing junior doctors in the United Kingdom or that experience and/or training can indeed influence an individual's outlook on the world.

\section{P-22 DECISION MAKING AROUND END OF LIFE CARE IN THE EMERGENCY DEPARTMENT}

Calvin Lightbody. NHS Lanarkshire, East Kilbride, UK

\subsection{6/bmjspcare-2017-00133.22}

Background Every day, Emergency Department (ED) doctors are confronted by the challenges associated with patients who are dying or who are nearing the end of life. Recognising that a patient is dying and identifying the need for a more palliative approach is crucial if the patient is to receive appropriate end of life care. Such decision making in the ED will also minimise harm to this patient group by avoiding treatments which would be futile, burdensome or contrary to the patient's wishes.
Method A communication tool was devised for use in the ED (named Hospital Anticipatory Care Plan - HACP). It allowed for the attending ED doctor to document what they felt were appropriate treatment options and also level of escalation in terms of any ITU/HDU referral. If considered that end of life care was most appropriate then this could also be indicated. Training in use of the tool was provided for medical and nursing staff of all grades.

Results One year after introduction of HACP, the tool is now commonly used in conjunction with DNACPR. An audit of cases admitted through the ED with HACP completed showed that in $73 \%$ of cases the patient died within 48 hours of admission - without being subjected to futile interventions or inappropriate escalation of care.

Conclusions Senior ED staff have fairly reliable clinical acumen in identifying patients who are on an end of life trajectory. Recognising that a patient is nearing the end of life makes it less likely that a patient will have a "bad death" ie, one that involves futile over treatment of their underlying condition while under treating their palliative needs.

\section{P-23 AN AUDIT OF INPATIENT HOSPICE METHADONE CONVERSION IN THE WEST MIDLANDS}

${ }^{1}$ Hannah Jennens, ${ }^{2}$ Sarah Freshwater, ${ }^{3}$ Christina Raddiffe. ${ }^{1}$ Compton Hospice, Wolverhampton; ${ }^{2}$ St Giles Hospice, Lichfield; ${ }^{3}$ St Mary's Hospice, Birmingham

\subsection{6/bmjspcare-2017-00133.23}

Background This audit was completed to review adherence to the current 2012 Pan Birmingham guidelines for the use of methadone in adults with pain in palliative care.

Methods Anonymised data collection tools were sent electronically to six hospices in the West Midlands. Data was collected retrospectively from controlled drugs record books over a 2 year period from $01 / 07 / 2013-30 / 06 / 2015$. The audit was limited to patients who were inpatient at the hospice and excluded those who were admitted already taking methadone on admission or taking it for an alternative reason eg, as part of a drug replacement programme.

Standards for the audit were derived from the current 2012 Pan Birmingham Guidance.

Results Results were received from five hospices. In total 28 patients were audited retrospectively over a 2 year period from July 2013 to June 2015. Ten patients were excluded. A protocol or guideline was in use in only 2 hospices $(40 \%)$. Of the 18 patients included in the audit methadone was commenced in 14 patients (78\%) due to poorly controlled pain despite other opiates. In one patient it was commenced due to tolerance to the analgesic effects of other opiates. In three patients methadone was commenced due to unacceptable side effects of other opiate medications. An ECG was performed prior to commencing methadone in only three patients. A variety of starting doses of methadone were used, most commonly $30 \mathrm{mg}$. Furthermore, breakthrough dosages of methadone prescribed were wide ranging from 2-30 mg.

Conclusions This audit shows that the current local guidelines are not always being adhered to. A wide variation in prescribing exists for both regular and PRN dosages of methadone. Additional areas for improvement have been identified including nursing training and QTc monitoring with pretreatment ECG. 